\title{
Estado actual de la gestión de residuos de construcción y demolición: limitaciones
}

\author{
State of the art on construction and demolition wastes \\ management: limitations
}

J. M. Morán del Pozo ${ }^{(*)}$, A. Juan Valdés( ${ }^{(*)}$, P. J. Aguado, M. I. Guerra, C. Medina

\section{RESUMEN}

El sector de la construcción ha crecido de forma exponencial en las últimas décadas y con él, la producción de residuos generados, estimándose esta en $2 \mathrm{~kg}$ por habitante y día, lo que representa una magnitud escalofriante. Para solucionar este problema la administración promueve comportamientos ecológicos respecto a la gestión de residuos de construcción y demolición (producción y posterior manejo y utilización de los mismos) a través de la redacción de normas que regulen el tema.

Estas normas presentan importantes carencias y limitaciones. Si estas carencias y limitaciones no se resuelven, el proceso encaminado a mejorar la sostenibilidad se interrumpe y las medidas tomadas hasta el momento se vuelven ineficaces.

Este trabajo pretende poner de manifiesto estos problemas a fin de evitarlos, de forma que todos los agentes implicados conozcan el importante papel que desempeñan en la correcta gestión.

\section{$113-96$}

Palabras Clave: Construcción, gestión, medio ambiente, residuos de construcción y demolición (RCDs), Sostenibilidad.

\section{SUMMARY}

Construction has grown exponentially in recent decades. This development has also brought large production of debris from construction and demolition. These wastes have reached the environment and generated a very important pollution in the landscape, soil and water. Equally it represents a loss of potential resources; because these wastes still have capacity to be recovered. Both central and regional government have published numerous standards, in recent years, to establish guidelines for a proper management, reuse and recycling of this debris.

This paper analyzes the current status of this legislation, their gaps and their degree of implementation.

Keywords: Construction, environment, management, residues from construction and demolition, sustainability. 


\section{ANTECEDENTES}

El sector de la construcción ha crecido de forma exponencial en las últimas décadas, y con él, la producción de residuos de construcción y demolición. Para dar una idea de la importancia de la generación de estos residuos cabe mencionar que el II Plan Nacional Integrado de Residuos (en adelante PNIR) (2008) (10) estima en $2 \mathrm{~kg}$ de residuos generados por habitante y día en España como media en los últimos años, lo que representa una magnitud de producción escalofriante.

España es el $5^{\circ}$ país europeo en producción de residuos de construcción y demolición reciclándose sólo el $5 \%$ de lo producido (del Rio, M) (2).

Estos residuos, en muchos casos, han ido a parar al medio ambiente generando importantes alteraciones del paisaje, contaminación de suelos y acuíferos y pérdida de recursos potenciales, ya que se desechan como residuos ciertos elementos provenientes de las obras que poseen todavía capacidad de ser valorizados a través de distintos procesos que están siendo analizados por numerosos investigadores, entre otros López, V. (6), Guerra, I. (4), el no aprovechamiento de estos elementos obliga a consumir recursos naturales, que de otra manera serían prescindibles, acentuando más el efecto negativo de esta actividad sobre el medio.

A medida que la sociedad del primer mundo alcanza cierto nivel de bienestar, valora recursos que mejoran su calidad de vida, sin los cuales las perspectivas de futuro se verían fuertemente comprometidas. Entre estos recursos, nos encontramos con el medio ambiente.

El importante problema generado por la actividad de la construcción ha provocado una fuerte alarma social que ha propiciado la aparición de normas legales que regulan la producción y gestión de los residuos de construcción y demolición y que obliga a la inclusión de un documento de carácter técnico en la fase de redacción de proyecto, con el que se pretende garantizar la aplicación real de la gestión de residuos de construcción y demolición.

La finalidad específica de esas normas implica:

- La reducción de la producción de residuos.

- La reutilización de aquellos residuos o elementos que así lo permitan.

-El reciclado de los residuos que no puedan reutilizarse.
- La valorización energética de los residuos que no puedan reciclarse.

- El depósito adecuado en vertedero de todo lo que no pueda valorizarse.

La aparición de estas normas podría frenar el deterioro del medio en el que nos vemos inmersos. Pero esto implica un cambio drástico en la mentalidad de todos los agentes implicados, siendo imprescindible su compromiso activo para lograr la sostenibilidad de la actividad constructiva, aportando así una pequeña contribución que, sumada a otras, permita acercarse a la sostenibilidad global.

El cambio que se pretende con la regulación de la gestión de residuos, es mucho más profundo que una mera obligación legal de redactar un documento engorroso, un simple trámite que justifique decisiones ya tomadas y que venga a engrosar el volumen de papel exigido en los proyectos.

Nuestra responsabilidad como proyectistas y miembros de esta sociedad, pasa por el conocimiento de la obligación moral de la necesidad ineludible de gestionar adecuadamente los residuos de construcción y demolición, como una aportación más que junto con otras, permita legar a nuestros hijos un mundo más habitable.

Esta obligación ética, no debiera requerir más respaldo que la necesidad de sostener la actividad de la construcción pero, por si alguien se lo cuestiona, la administración avanza hacia proporcionar ese respaldo a través de la promulgación de normas legales.

Hasta ahora, la opción más sencilla consistía en deshacerse de los residuos depositándolos en vertederos legales, en el mejor de los casos. Hoy en día, como ya hemos manifestado, esta opción no es sostenible y se pretende que en el futuro tampoco sea ventajosa desde un punto de vista económico, de manera que como profesionales de proyectos debemos ir preparándonos para el cambio.

Cabe destacar que la administración pretende desincentivar la mala gestión de residuos a través de la aplicación de medidas persuasivas, como las propuestas en la normativa de Castilla y León y mostradas aquí a modo de ejemplo (R.D. 54/2008) (1):

-Aumento del canon de vertido para residuos no segregados.

-Aplicación de fianzas a depositar por el contratista que sólo serán devueltas cuan- 
do la obra concluya habiendo gestionado adecuadamente los residuos.

Una vez manejados de manera sostenible los residuos en obra, se deben entregar a un gestor autorizado para su total valorización, es imprescindible que la Administración dote los medios necesarios para concluir el proceso de gestión ya que si no se completa, el sistema es inútil o al menos ineficaz.

Por tanto, la administración debe proporcionar herramientas que permitan concluir satisfactoriamente el proceso de gestión de residuos. Por ejemplo: en Castilla y León está previsto, en la normativa (R.D. 54/2008) (1), que se creen plantas de tratamiento de primer nivel, al menos en las capitales de provincia; de segundo nivel en otros núcleos con radio de acción de $35 \mathrm{~km}$ y zonas de almacenamiento o vertederos de residuos no peligrosos situados en otras zonas aisladas. Todo este entramado de gestores de residuos debe estar presente en número adecuado: sin él la aplicación del proceso es inviable.

El esquema de funcionamiento de una planta de tratamiento es el siguiente:

Inspección visual de la mezcla, pesado y determinación de la densidad estimada para establecer el tratamiento posterior del residuo. En esta zona se fijará el coste de la gestión.

Una vez el residuo es aceptado, pasa a la playa de descarga para la segregación por medios manuales o mecánicos de los distintos componentes.

Introducción en la línea de tratamiento y triaje compuesta por machacadora de mandíbulas, sistemas de cribado, trómeles, separadores y zona de acopio final. El material resultante pasa por un molino de impactos y un sistema de cribado para obtener áridos de diferentes granulometrías.

Estos áridos pueden ser empleados, entre otras obras, en:

- Infraestructuras

- Labores de conservación y mantenimiento

- Caminos agrícolas

Este tipo de plantas debe ser capaz de separar y en su caso tratar:

- Residuos peligrosos que serán entregados a gestores autorizados

-Maderas

- Hierros

-Envases metálicos

-Plásticos
- Hormigones, materiales cerámicos y bituminosos

- Cartones, papeles y mobiliario doméstico

-Vidrio

-Otros: botes de pinturas, aceites, barnices, fraguantes y aditivos.

Se podría concluir que, el proceso para sostenibilizar la actividad de la construcción, se debe desarrollar en tres fases:

\section{Fase 1: redacción del proyecto}

Implica la asunción de la sostenibilidad en el proceso de proyectar, asegurando la gestión adecuada de los residuos desde esta fase inicial con un diseño encaminado a la reducción de los residuos producidos, una planificación racional de la ejecución de la obra, del acopio de materiales y de la segregación de residuos.

Esta fase es una primera aproximación a la gestión de los residuos que permite cuantificar su coste y estimar las necesidades de espacio y medios, de manera que se garantice su desarrollo en la fase siguiente.

\section{Fase 2: ejecución de la obra}

En esta fase se debe adaptar el estudio de gestión de residuos a las características específicas de la obra y de la contrata que la desarrolle.

Fase 3: entrega de los residuos generados al gestor que deberá concluir el proceso

En esta fase el gestor deberá tratar el residuo convenientemente para permitir su reutilización, reciclado, valorización energética o deposito conveniente sin provocar un efecto negativo sobre el medio.

Si cualquiera de las tres fases falla, el proceso se interrumpe, por lo que es imprescindible que todos los implicados en cada una de ellas actúen conforme a los principios de sostenibilidad.

\section{ESTUDIO DE GESTIÓN DE RESIDUOS}

El Real Decreto 105/2008 establece la obligación de incluir en el proyecto de obra un "estudio de gestión de residuos". Debemos entender, que este estudio es una estimación de los residuos que se van a producir en la obra y su pretensión, es ser una herramienta para incorporar la gestión de los residuos desde el inicio del proyecto para acercarse a la sostenibilidad de la actividad constructiva, estimando los medios y los costes necesarios para llevarla a cabo, aunque se asuma la necesidad posterior de adaptar el proceso de gestión de residuos, a las circunstancias reales de cada obra. 
Esta necesidad de modificar en parte la información recogida en el estudio, no invalida su utilidad que, como hemos mencionado, va más allá de cuantificar con exactitud los parámetros relacionados con los residuos.

Es importante redactar este documento de forma específica para cada obra, incorporando las mejores soluciones de diseño que optimicen la eficacia del mismo.

El contenido del estudio habrá de adaptarse a las exigencias del artículo 4.1.a del Real Decreto 105/2008 y recogido a continuación:

a) Estimación de la cantidad expresada en toneladas y en metros cúbicos de los residuos de construcción y demolición, codificados según la lista europea recogida en la Orden MAM/304/2002.

b) Medidas para la prevención de residuos en la obra objeto del proyecto.

c) Las operaciones de reutilización, valorización o eliminación a que se destinarán los residuos que se generan en la obra.

d) La medidas para la separación de los residuos en obra.

e) Los planos de las instalaciones previstas para el almacenamiento, manejo, separación y, en su caso, otras operaciones de gestión de los residuos de construcción y demolición dentro de la obra.

f) El pliego de prescripciones técnicas particulares del proyecto, en relación con el almacenamiento, manejo, separación y, en su caso, otras operaciones de gestión de los residuos de construcción y demolición dentro de la obra.

g) Una valoración del coste previsto de la gestión de los residuos de construcción y demolición que formará parte del presupuesto del proyecto en capítulo independiente.

Los puntos desde la a) hasta la d) podrían constituir la memoria del documento.

A continuación se dan ciertas aclaraciones que pueden facilitar la redacción de alguna de las partes citadas.

Apartado a:

Se debe realizar, en primer lugar, la identificación de los distintos elementos que componen los escombros elaborando una lista de residuos desagregados, para ellos se utiliza la lista europea de residuos publicada en la Orden MAM/304/2002 (BOE DEL 19 DEL 02 DE 2002) (7).
En ella los distintos elementos vienen codificados a través de seis dígitos, los dos primeros corresponden al capítulo, el caso de los residuos de construcción y demolición, el dígito indicativo de este capítulo es el 17. Los dos dígitos siguientes identifican grupos de residuos de una clase determinada, por ejemplo 01 para el grupo de "hormigón, ladrillo, tejas y materiales", 02 para el grupo de "maderas, vidrio y plástico", etc. Los dos últimos dígitos definen el elemento concreto de que se trata.

Cabe destacar que, al consultar el capítulo 17 de la lista europea de residuos, se observa una falta de continuidad en la numeración achacable a la omisión de algunos capítulos y elementos, si bien parte de estas carencias se subsanaron en una modificación posterior publicada en el BOE del 12 de marzo de 2002, todavía quedan algunos grupos sin definir.

Una vez identificados los elementos se debe estimar la cantidad de cada uno de ellos, que se va a producir, para ellos. Las distintas comunidades autónomas proponen métodos que permiten realizar la cuantificación aproximada y que quedan recogidos en la normativa que publica cada comunidad.

En esencia, los métodos propuestos son idénticos en las distintas comunidades autónomas. La única variación consiste en la magnitud de los parámetros utilizados.

A continuación se propone, a modo de ejemplo, un esquema de "estudio de gestión de residuos" adaptado a las exigencias del Real Decreto 105/2008 (9) y que utiliza, como procedimiento de cálculo para la determinación de los parámetros, el recogido en el Decreto 54/2008:"Plan regional de residuos de construcción y demolición de Castilla y León 2008-2010" (1).

a) Determinación de los volúmenes de tierra y de escombros desagregados:

La determinación del volumen de tierra producido en la excavación se extrae del presupuesto del proyecto (mediciones).

El cálculo orientativo del volumen de escombros agregados que se genera en obra se suele realizar utilizando coeficientes que permiten estimar a partir de la superficie construida, demolida o reformada, el volumen de escombros producidos. Estos coeficientes han sido establecidos a través de estudios estadísticos de obras reales (ITEC 2000) (5) y viene a significar la altura de escombros generada por cada metro cuadrado de obra.

En el caso de Castilla y León, para obra nueva, se estima que por cada metro cuadrado de obra construida se generan $12 \mathrm{~cm}$ de al- 
tura de escombros. Por lo tanto, el coeficiente para obra nueva es:

$$
\mathrm{C}_{\mathrm{O} . \mathrm{N}}=0,120 \mathrm{~m}^{3} / \mathrm{m}^{2} \text { construido }
$$

De forma análoga, para obra de reforma, el coeficiente es:

$$
\mathrm{C}_{\text {O.R. }}=0,4892 \mathrm{~m}^{3} / \mathrm{m}^{2} \text { reformado }
$$

Y para obra de demolición, el coeficiente es:

$$
\mathrm{C}_{\text {O.D. }}=0,8583 \mathrm{~m}^{3} / \mathrm{m}^{2} \text { demolido }
$$

Una vez conocemos el volumen de escombros producidos, se puede establecer el peso a partir de la densidad media. El dato orientativo que se propone en Castilla y León es $1,4 \mathrm{t} / \mathrm{m}^{3}$. Una vez estimada la densidad se debe determinar la cantidad de escombros de cada tipo que se producen.

La composición de los residuos de construcción y demolición es muy heterogénea estando formados, en su mayor parte, por mezcla de áridos, hormigones, piedra, materiales cerámicos, gravas y arenas y, en menor medida, por maderas, metales, vidrio, papel, plásticos y otros elementos.

EI PNIR (10) establece, como composición típica de los residuos, la recogida en la Tabla 1, basada en estudios estadísticos realizados en obras reales. Destacar que esta tabla coincide con la propuesta en la normativa específica de Castilla y León.

En ella se dan los porcentajes en peso que representa cada fracción desagregada en el total de los escombros. Cabe destacar que ni en la norma de Castilla y León, ni en el PNIR se esclarece si los porcentajes de residuos mostrados en la tabla son porcentajes en peso o en volumen, pero tras el análisis de la normativa de otras comunidades autónomas (normativa de la Comunidad Autónoma de Madrid (8)) se puede llegar a la conclusión de que se trata de porcentajes en peso.

Utilizando esos porcentajes se determina el peso de los distintos elementos que constituyen los residuos. El siguiente paso sería establecer el volumen que ocupan, para lo cual es necesario conocer las densidades de los distintos residuos desagregados (Tabla2). Estos datos son difíciles de estimar y constituyen una de las carencias más importantes de la actual normativa, en donde se exige la determinación del volumen de estos elementos y no se proporcionan las densidades, indispensables para la estimación.

Tras la consulta a distintas fuentes, basadas en datos estadísticos ITEC (2000) (5), se pueden establecer las siguientes densidades orientativas (ver tabla 2):
Conocidas las densidades y los pesos de los residuos desagregados, calculamos el volumen que ocupará cada elemento, lo cual nos permitirá determinar más adelante, los contenedores necesarios para la segregación de los residuos in situ.

b) Medidas para la reducción de emisiones:

- En fase de proyecto, se deben adoptar todas las soluciones de diseño que permitan reducir la cantidad de residuos (material sobrante).

Tabla 1

\begin{tabular}{|c|c|c|}
\hline MATERIALES & $\%$ & DE COMPOSICIÓN \\
\hline FRACCIÓN PÉTREA & & 75 \\
\hline Ladrillos, azulejos y otros cerámicos & & 54 \\
\hline Hormigón & & 12 \\
\hline Piedra & & 5 \\
\hline Arena, grava y otros áridos & & 4 \\
\hline RESTO & & 25 \\
\hline Madera & & 4 \\
\hline Vidrio & & 0,5 \\
\hline Plástico & & 1,5 \\
\hline Metales & & 2,5 \\
\hline Asfalto & & 5 \\
\hline Yeso & & 0,2 \\
\hline Basura & & 7 \\
\hline Papel & & 0 \\
\hline Otros & & 4 \\
\hline
\end{tabular}

Composición en peso de los RCD producidos en obras de construcción. Plan Nacional de RCD (año)

-En la fase de ejecución de obra, se deben adoptar las prácticas constructivas de manejo y gestión de materiales que permitan reducir la cantidad de residuos.

De manera innovadora se están desarrollando líneas de investigación que analizan la valorización de los residuos provenientes de esta actividad, transformando ciertos desechos en materiales útiles; López, V. (6); Guerra, I. (4).

c) Operaciones de reutilización, valoración o eliminación adoptadas:

Siempre teniendo en cuenta la operación más adecuada en función del tipo de residuo y de las posibilidades reales (dependiendo de las características del gestor presente en la zona) de gestión. 
Tabla 2

Densidades medias de los residuos desagregados

\begin{tabular}{|l|c|}
\hline \multicolumn{1}{|c|}{ MATERIALES } & Densidad $\left(\mathrm{t} / \mathrm{m}^{3}\right)$ \\
\hline \multicolumn{1}{|c|}{ FRACCIÓN PÉTREA } & 1,5 \\
\hline Ladrillos, azulejos y otros cerámicos & 1,5 \\
\hline Hormigón & 1,5 \\
\hline Piedra & 1,5 \\
\hline Arena, grava y otros áridos & \\
\hline \multicolumn{1}{|c|}{ RESTO } & 0,6 \\
\hline Madera & 1,5 \\
\hline Vidrio & 0,9 \\
\hline Plástico & 1,5 \\
\hline Metales & 1,3 \\
\hline Asfalto & 1,2 \\
\hline Yeso & 0,9 \\
\hline Basura & 0,9 \\
\hline Papel & 0,5 \\
\hline Otros & \\
\hline
\end{tabular}

Las propias obras pueden ser receptoras de materiales provenientes, del reciclado de las mismas o de la industria, López, V. (6); Guerra, I. (4); Valdés, A.J. (11); González, B. (3) transformándolos en elementos valorizables, que han sido tradicionalmente considerados residuos sin utilidad y cuya acumulación suponía un importante problema ambiental.

En este punto nos encontramos con otro importante problema y es la presencia completamente heterogénea de gestores que traten estos residuos y la dificultad por lo tanto de dar la adecuada valorización a los mismos.

Todavía existen numerosas provincias en donde no encontramos gestores que valoricen los residuos por lo que a día de hoy el proceso es ineficaz, esto implica que aunque se trate de optimizar las fases de proyecto y de ejecución, es imprescindible que la administración impulse la presencia de gestores que concluyan el proceso.

d) Segregación de los residuos:

Una vez conocemos el volumen de residuos desagregados calculado en el apartado a) y las operaciones de valorización y eliminación que vamos a llevar a cabo, estamos en condiciones de establecer las necesidades de contenedores y otros medios para procurar la segregación en obra.

El contenido del estudio a partir de este punto será planos, pliego y presupuesto.

e) Los planos que se incluyan en el proyecto deben contener detalles acerca de ubicación de contenedores, presencia de otros elementos para la gestión de los residuos, acopio detallado de materiales.

La distribución en planta de los detalles que permiten gestionar los residuos se realizará buscando el diseño óptimo y la máxima funcionalidad de acuerdo a las características de la obra y del entorno. La información correspondiente a este apartado debería recogerse en el documento número 2 del proyecto, a fin de facilitar su aplicación en la fase de obra.

f) El pliego de condiciones donde se recojan las obligaciones y derechos de las distintas partes implicadas en la gestión de residuos. La información correspondiente a este apartado debería recogerse en el documento número 3 del proyecto, a fin de favorecer su cumplimiento.

g) El presupuesto, es el lugar en donde se estiman los gastos derivados de la gestión correcta de los residuos, su inclusión en el estudio y posteriormente en el documento número 4 garantiza su aplicación real.

\section{CONCLUSIONES}

A continuación, se exponen las carencias más relevantes que se han encontrado tras este análisis:

\section{Fase 1: Redacción del proyecto}

La normativa es imprecisa y los métodos de cálculo carecen de algunos datos imprescindibles para determinar los parámetros necesarios y exigidos por la normativa actual, para que quede definida la correcta gestión.

La posibilidad de "eludir" la obligación de segregación de residuos anima a la realización del proceso de cálculo de la cantidad de residuos generados de abajo a arriba en lugar de al revés, es decir preguntándose previamente cuantos residuos tengo que producir para estar exento de la segregación, y amañando los cálculos para ajustarse a esos límites.

Existe una falta clara de concienciación del proyectista y del promotor:

- El promotor ve la obligación de gestionar los residuos como un requisito costoso cuyos beneficios no repercuten directamente sobre él.

- El proyectista ve la obligación de gestionar los residuos como un trámite engorroso que aumenta el trabajo a realizar en el proyecto y que hay que "vadear" de cualquier forma a través de la inclusión de un estudio genérico y en absoluto adaptado 
a las peculiaridades específicas de la obra y por lo tanto inútil.

Fase 2: Ejecución de la obra

Existe una falta de concienciación de la contrata que busca en muchas ocasiones eludir la obligación y solventarla de la manera más cómoda, no de la forma más eficaz y sostenible

Fase 3: Entrega de los residuos generados al gestor que deberá concluir el proceso

La administración debe proporcionar los medios que faciliten la gestión, es decir gestores próximos a todas las provincias y en número suficiente, cosa que actualmente no existe.

Además se deben articular medidas que desincentiven la mala gestión.

\section{Agradecimientos}

Este trabajo de investigación ha sido parcialmente realizado gracias a los fondos del proyecto "Hormigones reciclados eco-eficientes con fracción pétrea cerámica de residuos de construcción y demolición", subvencionado por la Universidad de León.

\section{BIBLIOGRAFÍA}

(1) Decreto 54/2008, de 17 de julio, por el que se aprueba el Plan Regional de Ámbito Sectorial de Residuos de la Construcción y Demolición de Castilla y León. BOCYL, suplemento al n 141, 1-128, 23 de julio (2008).

(2) Del Rio, M.; Izquierdo, P.; Salto, I.; Cruz, J. S.: "La regulación jurídica de los residuos de construcción demolición (RCD) en España. El caso de la Comunidad de Madrid". Informes de la Construccion, Vol. 62 no 517 (2010), pp. 81-86. doi: 10.3989/ic.08.059.

(3) González, B.; Llamas, B.; Juan A. et al.: "Tests on concrete containing cork powder admixtures", Materiales de Construcción, Vol. 57 nº 286 (2007), pp. 83-90.

(4) Guerra, I.; Vivar, I.; Llamas, B.; et al.: "Eco-efficient concretes: The effects of using recycled ceramic material from sanitary installations on the mechanical properties of concrete", Waste Management, Vol. 29 n $^{\circ} 2$ (2009), pp. 643-646.

(5) Instituto de Tecnología de la Construcción de Cataluña (ITeC). "Situación actual y perspectivas de futuro de los residuos de la construcción", p. 41, ITeC, Cataluña (España), 2000.

(6) López, V.; Llamas, B.; Juan, A.; et al.: "Eco-efficient concretes: Impact of the use of white ceramic powder on the mechanical properties of concrete", Biosystems Engineering, Vol. 96 n 4 (2007), pp. 559-564.

(7) Orden MAM/304/2002, de 8 de febrero, por la que se publican las operaciones de valorización y eliminación de residuos y la lista europea de residuos, BOE, n 43, 6494-6515, 19 de febrero (2002).

(8) ORDEN 2726/2009, de 16 de julio, por la que se regula la gestión de los residuos de construcción y demolición en la Comunidad de Madrid. BOCM, nº 186, 4-12, 7 de agosto (2009).

(9) Real Decreto 105/2008, de 1 de febrero, por el que se regula la producción y gestión de los residuos de construcción y demolición. BOE, n 38, 7724 - 7730, 13 de febrero (2008).

(10) Resolución de 20 de enero de 2009, de la Secretaria de Estado de Cambio Climático, por la que se pública el Acuerdo del Consejo de Ministros por el que se aprueba el Plan Nacional Integrado de Residuos para el periodo 2008-2015. BOE, no 49, 19893-20016, 26 de febrero (2009).

(11) Valdes, A. J.; Martinez, C. M.; Romero, M. I. G.; et al.: "Re-use of construction and demolition residues and industrial wastes for the elaboration or recycled eco-efficient concretes", Spanish Journal Of Agricultural Research, Vol. 8 n 1 (2010), pp. 25-34 